\title{
Searches for New Physics with the TREK Detector
}

\author{
Steffen Strauch ${ }^{* \dagger}$ for the TREK Collaboration \\ University of South Carolina \\ E-mail: strauch@sc.edu
}

Experiment E36 at the Japan Proton Accelerator Research Complex (J-PARC) will study the decay of stopped kaons to search for new physics beyond the Standard Model. In particular, the experiment aims to provide the most stringent test of lepton universality to date in a measurement of the ratio of the $K_{e 2}$ and $K_{\mu 2}$ decay widths, and will simultaneously search for light new particles, such as a heavy sterile neutrino or a dark photon. The experimental setup is based on the upgraded detector of KEK experiment E246 and includes a large-acceptance toroidal spectrometer for tracking charged particles with high resolution, combined with a photon calorimeter with large solid angle. The current status of the upcoming experiment and the planned measurements will be presented.

XIIth International Conference on Heavy Quarks \& Leptons 2014

25-29 August 2014

Schloss Waldthausen, Mainz, Germany

\footnotetext{
* Speaker.

${ }^{\dagger}$ Supported in parts by the U.S. National Science Foundation: PHY-1205782.
} 


\section{Overview and Instrumentation}

Despite its overwhelming success, the Standard Model (SM) of particle physics is incomplete. It does not account for neutrino oscillations and cold dark matter nor does it explain the matterantimatter asymmetry in the universe. Kaon decays are powerful tools to search for new physics beyond the Standard Model. The TREK Collaboration is preparing two experiments, E36 [1] and E06 [2], to study the decay of stopped kaons with high-intensity kaon beams at the Japan Proton Accelerator Research Complex (J-PARC). The decays of interest include $K^{+} \rightarrow e^{+} v[\gamma]$ and $K^{+} \rightarrow$ $\mu^{+} v[\gamma]$ to determine the ratio of the $K_{e 2}$ and $K_{\mu 2}$ decay widths, as well as $K^{+} \rightarrow \pi^{+} A^{\prime} \rightarrow \mu^{+} v e^{+} e^{-}$ and $K^{+} \rightarrow \mu^{+} v A^{\prime} \rightarrow \pi^{+} e^{+} e^{-}$in the search for lepton flavor violating light neutral bosons.

The experiments will utilize the 12-sector superconducting iron-core toroidal spectrometer of E246 [3] with upgraded instrumentation. A schematic view of the TREK detector for experiment E36 after upgrade from the KEK E246 apparatus is shown in Fig. 1. A collimated mixed $K^{+} / \pi^{+}$

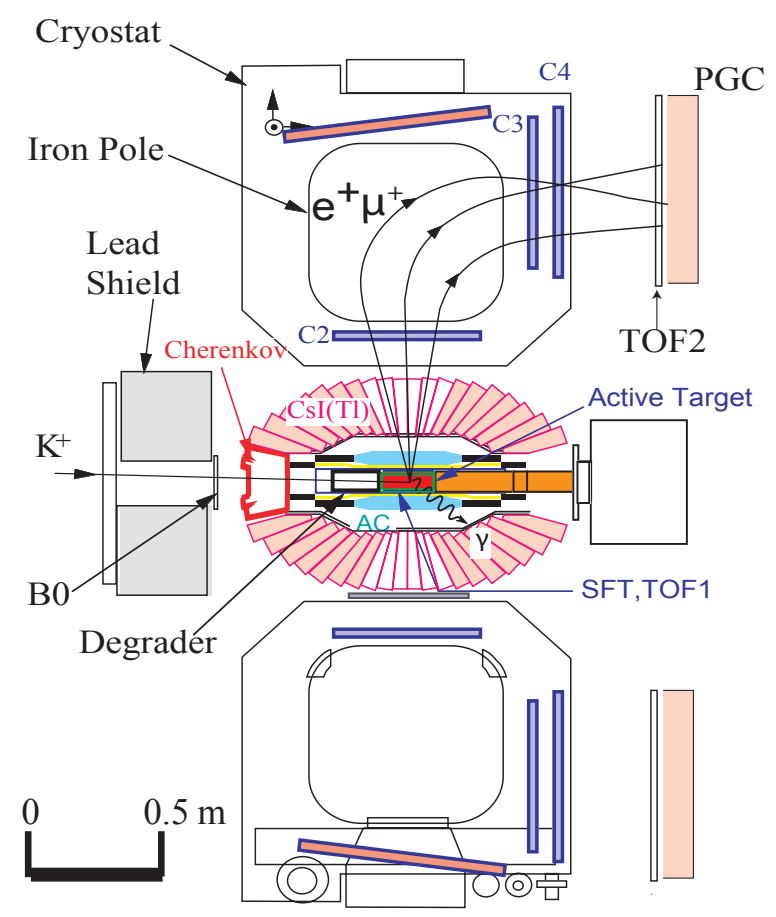

Figure 1: Schematic view of the TREK detector for experiment E36 [1] after upgrade from the KEK E246 apparatus. Figure courtesy of K. Horie.

beam with a momentum of $800 \mathrm{MeV} / c$ from the of K1.1BR beam line passes through a degrader system and stops in a position-sensitive fiber target in the center of the setup. The Cherenkov detector, upstream of the target, identifies beam kaons. Charged kaon-decay particles are tracked by a Spiral Fiber Tracker (SFT) and three multi-wire proportional chambers C2, C3, and C4. The curvature of the track in the spectrometer determines the particle's momentum. Photons, positrons, and electrons from the kaon decays are detected in the $\mathrm{CsI}(\mathrm{Tl})$ electromagnetic calorimeter. The time-of-flight counters (TOF1 and TOF2), the aerogel Cherenkov detector (AC) close to the target, 
and the lead glass Cherenkov detectors (PGC) at the exit of the spectrometer are used for $e / \mu$ particle separation.

\section{Measurements}

\subsection{Ratio of the $K_{e 2}$ and $K_{\mu 2}$ decay widths}

Experiment E36 aims to provide the most stringent test of lepton universality to date in a measurement of the ratio of the $K_{e 2}$ and $K_{\mu 2}$ decay widths,

$$
R_{K}=\frac{\Gamma\left(K^{+} \rightarrow e^{+} v_{e}[\gamma]\right)}{\Gamma\left(K^{+} \rightarrow \mu^{+} v_{\mu}[\gamma]\right)}
$$

The ratio is to first order free of hadronic uncertainties as the hadronic form factor, $f_{K}$, cancels in the ratio. The Standard-Model value of $R_{K}$ can be calculated with high precision, $R_{K}^{S M}=2.477(1) \times$ $10^{-5}$ [4], and provides a clean basis to detect or constrain non-standard physics, such as nonuniversal lepton couplings. In a model, where charged Higgs exchange is the dominant SUSY contribution, an increase of $R_{K}$ by $\mathscr{O}(1 \%)$ could be possible [5]. $R_{K}$ is also sensitive to neutrino mixing parameters with SM extensions involving a fourth generation of quarks and leptons [6] or sterile neutrinos [7]. New physics that differentiates between lepton species has been discussed, e.g. $[8,9]$, as possible explanation for the proton-radius puzzle [10]. The proton-radius puzzle is the significant disagreement between extractions of the proton radius from muonic hydrogen data on the one hand, and from electronic hydrogen or elastic electron-proton scattering on the other hand.

Figure 2 shows recent results for $R_{K}$ from the $\operatorname{KLOE}$ [11] and NA62 [12, 13, 14] Collaborations with relative uncertainties of $1.3 \%$ and $0.4 \%$ respectively. Also shown are the projected uncertainties of E36. The width of the vertical bar at zero indicates the relative uncertainty of the $\mathrm{SM}$ value, $R_{K}^{S M}$. The proposed experiment E36 is a complementary measurement with a stopped

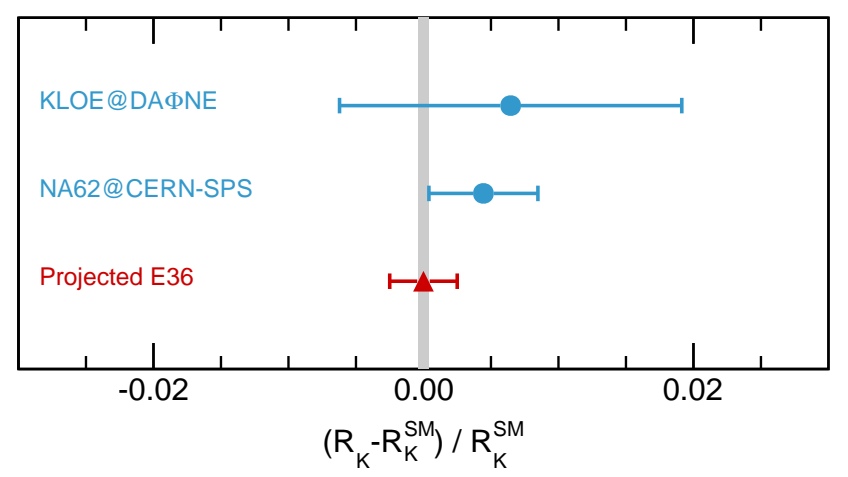

Figure 2: Relative deviations of recent high-precision measurements of $R_{K}$ from the standard-model value $R_{K}^{S M}$. Experimental results (full circle) are from the KLOE [11] and NA62 [12, 13, 14] collaborations. These experiments are ongoing. The projected uncertainty of E36 [1] (triangle) is shown at the standard-model value.

$K^{+}$beam and has systematic uncertainties very different from those of KLOE and NA62. The 
$K_{e 2}$ and $K_{\mu 2}$ events will be identified by the charged lepton momenta, $p_{e}=247 \mathrm{MeV} / c$ and $p_{\mu}=236 \mathrm{MeV} / c$, as determined in the spectrometer. Excellent $e / \mu$ particle separation for the decay products is required for this measurement. The combined information from the TOF, PGC, and $\mathrm{AC}$ counters reduce the probability of $\mu^{+}$mis-identification as $e^{+}$to less than $10^{-6}$; together with the particle momentum information the probability is further reduced to $10^{-8}$. The CsI(Tl) calorimeter will serve as photon detector for the radiative processes. The $R_{K}$ value is then derived from the number of the identified kaon decays, $N\left(K_{e 2[\gamma]}\right)$ and $N\left(K_{\mu 2[\gamma]}\right)$, including those with the production of internal bremsstrahlung but excluding structure-dependent contributions, and from the detector acceptances, $\Omega$,

$$
R_{K}=\frac{N\left(K_{e 2}\right)}{N\left(K_{\mu 2}\right)} \frac{\Omega\left(K_{\mu 2}\right)}{\Omega\left(K_{e 2}\right)} .
$$

The stopped kaon decay, the rotational symmetry of the toroidal spectrometer, and the ratio of the detector acceptances help reduce the systematic uncertainties. The total expected uncertainty in the measurement of $R_{K}$ is expected to be $0.25 \%$.

\subsection{Search for lepton flavor violating light neutral bosons}

A possible extension of the Standard Model includes an additional $U(1)_{D}$ gauge group manifesting itself in a massive gauge boson $A^{\prime}$, "dark photon", in the $\mathrm{MeV}$ to $\mathrm{GeV}$ mass range. Recent searches for dark photons have been performed by the KLOE-2 [15], APEX [16], Mainz A1 [17], and BABAR [18] collaborations and put upper limits on the coupling of the massive gauge boson to charged particles.

Rare kaon decay can provide constraints on the $A^{\prime}$ parameters [19]. A total of $2.5 \times 10^{11}$ kaons are expected to be stopped in the E36 target. The observation of the processes $K^{+} \rightarrow \mu^{+} v_{\mu} A^{\prime}$ and $K^{+} \rightarrow \pi^{+} A^{\prime}$ is a possible signal from the dark sector. The $A^{\prime}$ particle could be identified in the invariant-mass distribution, $m\left(e^{+} e^{-}\right)$, through its decay, $A^{\prime} \rightarrow e^{+} e^{-}$. The $e^{+} e^{-}$pair will be detected in the $\mathrm{CsI}(\mathrm{Tl})$ calorimeter and allows for a full reconstruction of the $\mu^{+} v e^{+} e^{-}$and $\pi^{+} e^{+} e^{-}$final states. A peak in the $m\left(e^{+} e^{-}\right)$data on top of a smooth, calculable background from the radiative $K^{+} \rightarrow \mu^{+} v_{\mu} e^{+} e^{-}$kaon decay corresponds to the mass of the $A^{\prime}$, if it exists; the width of the peak is determined by the detector's resolution. The calculated full phase-space value for the branching ratio for that radiative background channel is $\approx 2.49 \times 10^{-5}$ [20] and corresponds to about 200,000 events in the expected E36 data sample.

Present estimates [21] show that under the assumption of universal coupling results from E36 will be mostly complementary to existing data. However, an explanation of the proton-radius puzzle and the muon's anomalous magnetic moment involving new particles must include larger couplings to muons than electrons and violate lepton universality. Such models predict striking and observable peaks in the $m\left(e^{+} e^{-}\right)$distributions for E36 [22]. TREK E36 can rule out any new physics explanation of the proton radius puzzle involving light bosons with preferred couplings to muons.

\subsection{Search for sterile neutrinos}

The Neutrino Minimal Standard Model $(v \mathrm{MSM})$ is an example of a renormalizable extension of the SM that contains three right-handed (sterile) neutrinos with masses below the electroweak 
scale [23]. The lightest of the three sterile neutrinos is a dark-matter candidate, the masses of the heavier two sterile neutrinos are degenerate in the $v \mathrm{MSM}, M_{1}<M_{2} \approx M_{3}$. If the sterile neutrino is lighter than the kaon, it can give rise to leptonic and semi-leptonic decays with relatively large branching ratios. Experiment E36 is not optimized for the sterile-neutrino search. However, heavy sterile neutrinos, $N$, can be identified in the E36 data sample through narrow peaks in the lepton momentum distributions from $K^{+} \rightarrow e^{+} N$ and $K^{+} \rightarrow \mu^{+} N$ decays. The search could be optimized with an improved photon veto.

\subsection{Transverse muon polarization in $K^{+} \rightarrow \pi^{0} \mu^{+} v$}

Experiment E06 aims to improve the limit on the magnitude of the $T$-odd transverse muon polarization, $P_{T}$, decays set by KEK experiment E246 [24] by a factor of 20. The muon polarization is particularly useful for searches of $T$-violation effects from non-SM physics as it vanishes in the Standard Model at the tree level and $T$-odd contributions from final-state interactions are very small, $<10^{-5}$ [25]. Thus, any measurement of $\left|P_{T}\right|$ in the experimentally reachable region of $10^{-3}$ to $10^{-4}$ would unambiguously imply physics beyond the Standard Model. The experiment requires an active muon polarimeter at the exit of the spectrometer and an intense kaon beam. The experiment is considered when higher beam power is available at J-PARC.

\section{Outlook}

The TREK collaboration is pursuing searches for new physics in the study of the decay of stopped kaons with two experiments, E06 and E36, at J-PARC. The program includes the search for lepton universality violation in a measurement of the ratio of the $K_{e 2}$ and $K_{\mu 2}$ decay widths and the search for dark light in the $K^{+} \rightarrow \mu^{+} v A^{\prime} \rightarrow \mu^{+} v e^{+} e^{-}$and $K^{+} \rightarrow \pi^{+} A^{\prime}$ reactions. E36 is currently being installed and it is scheduled to run in 2015. The search for $T$ violation in kaon decays through the measurement of the transverse muon polarization in the $K^{+} \rightarrow \pi^{0} \mu^{+} v_{\mu}$ reaction, experiment E06, requires the active muon polarimeter and maximum kaon-beam intensities and will run later.

\section{References}

[1] J-PARC Experiment E36, Measurement of $\Gamma\left(K^{+} \rightarrow e^{+} v\right) / \Gamma\left(K^{+} \rightarrow \mu^{+} v\right)$ and Search for heavy sterile neutrinos using the TREK detector system, M. Kohl and S. Shimizu, spokespersons.

[2] J-PARC Experiment E06, Measurement of T-violating Transverse Muon Polarization in $K^{+} \rightarrow \pi^{0} \mu^{+} v$ Decays, J. Imazato and M. Kohl, spokespersons.

[3] M. Abe et al., Apparatus for a search for T violating muon polarization in stopped kaon decays, Nucl. Instrum. Meth. A506 (2003) 60, hep-ex/ 0302001.

[4] V. Cirigliano and I. Rosell, Two-loop effective theory analysis of $\pi(K) \rightarrow e \bar{v}_{e}[\gamma]$ branching ratios, Phys. Rev. Lett. 99 (2007) 231801, 0707 . 3439.

[5] A. Masiero, P. Paradisi, and R. Petronzio, Probing new physics through $\mu-e$ universality in $K \rightarrow \ell v$, Phys. Rev. D 74 (2006) 011701, hep-ph / 0511289.

[6] H. Lacker and A. Menzel, Simultaneous Extraction of the Fermi constant and PMNS matrix elements in the presence of a fourth generation, JHEP 1007 (2010) 006, 1003.4532. 
[7] A. Abada, D. Das, A. Teixeira, A. Vicente, et al., Tree-level lepton universality violation in the presence of sterile neutrinos: impact for $R_{K}$ and $R_{\pi}$, JHEP 1302 (2013) 048, 1211.3052.

[8] B. Batell, D. McKeen, and M. Pospelov, New Parity-Violating Muonic Forces and the Proton Charge Radius, Phys. Rev. Lett. 107 (2011) 011803, 1103.0721.

[9] C. E. Carlson and B. C. Rislow, New Physics and the Proton Radius Problem, Phys.Rev. D86 (2012) $035013,1206.3587$.

[10] R. Pohl, R. Gilman, G. Miller, and K. Pachucki, Muonic hydrogen and the proton radius puzzle, Ann.Rev.Nucl.Part.Sci. 63 (2013) 175, 1301 . 0905.

[11] F. Ambrosino et al., Precise measurement of $\Gamma(K \rightarrow e v(\gamma)) / \Gamma(K \rightarrow \mu v(\gamma))$ and study of $K \rightarrow e v \gamma$, Eur. Phys. J. C64 (2009) 627, 0907 . 3594.

[12] E. Goudzovski, Lepton flavour universality test at the CERN NA62 experiment, Nucl. Phys. Proc. Suppl. 210-211 (2011) 163, 1008 . 1219.

[13] C. Lazzeroni et al., Test of Lepton Flavour Universality in $\mathrm{K}^{+} \rightarrow l^{+} v$ Decays, Phys. Lett. $\mathbf{B 6 9 8}$ (2011) 105, 1101.4805.

[14] C. Lazzeroni et al., Precision Measurement of the Ratio of the Charged Kaon Leptonic Decay Rates, Phys. Lett. B719 (2013) 326, 1212 . 4012.

[15] F. Archilli, D. Babusci, D. Badoni, I. Balwierz, et al., Search for a vector gauge boson in phi meson decays with the KLOE detector, Phys. Lett. B706 (2012) 251, 1110 . 0411.

[16] S. Abrahamyan, Z. Ahmed, K. Allada, D. Anez, et al., Search for a New Gauge Boson in Electron-Nucleus Fixed-Target Scattering by the APEX Experiment, Phys. Rev. Lett. 107 (2011) 191804.

[17] H. Merkel, P. Achenbach, C. Ayerbe Gayoso, J. C. Bernauer, et al., Search for Light Gauge Bosons of the Dark Sector at the Mainz, Microtron, Phys. Rev. Lett. 106 (2011) 251802.

[18] B. Aubert, Y. Karyotakis, J. P. Lees, V. Poireau, et al., Search for Dimuon Decays of a Light Scalar Boson in Radiative Transitions $\Upsilon \rightarrow \gamma A^{0}$, Phys. Rev. Lett. 103 (2009) 081803.

[19] T. Beranek and M. Vanderhaeghen, Constraints on the Dark Photon Parameter Space from Leptonic Rare Kaon Decays, Phys. Rev. D87 (2013) 015024, 1209.4561.

[20] J. Bijnens, G. Ecker, and J. Gasser, Radiative Semileptonic Kaon Decays, Nucl. Phys. B396 (1993) 81, hep-ph/9209261.

[21] M. Kohl and P. Monaghan, private communication (2014).

[22] C. E. Carlson and B. C. Rislow, Constraints to new physics models for the proton charge radius puzzle from the decay $K^{+} \rightarrow \mu^{+}+v+e^{-}+e^{+}$, Phys.Rev. D89 (2014) 035003, 1310.2786.

[23] L. Canetti, M. Drewes, and M. Shaposhnikov, Sterile Neutrinos as the Origin of Dark and Baryonic Matter, Phys. Rev. Lett. 110 (2013) 061801, 1204 . 3902.

[24] M. Abe, M. Aliev, V. Anisimovsky, M. Aoki, et al., Search for T-violating Transverse Muon Polarization in the $K^{+} \rightarrow \pi^{0} \mu^{+} v$ Decay, Phys. Rev. D 73 (2006) 072005.

[25] V. Efrosinin, I. Khriplovich, G. Kirilin, and Y. Kudenko, Transverse muon polarization in $K^{ \pm} \rightarrow \pi^{0} \mu^{ \pm} v$ decay induced by the two photon final state interaction, Phys. Lett. B493 (2000) 293, hep-ph/0008199. 